\title{
Anabases
}

ANABASES Traditions et réceptions de l'Antiquité

\section{Daniel Barbu, Nicolas Meylan, Youri Volokhine (éd.), Mondes Clos. Les îles}

\section{Élodie Guillon}

\section{(2) OpenEdition}

1 Journals

\section{Édition électronique}

URL : http://journals.openedition.org/anabases/5735

DOI : 10.4000/anabases.5735

ISSN : 2256-9421

\section{Éditeur}

E.R.A.S.M.E.

\section{Édition imprimée}

Date de publication : 10 novembre 2016

Pagination : 331-332

ISSN : 1774-4296

\section{Référence électronique}

Élodie Guillon, « Daniel Barbu, Nicolas Meylan, Youri Volokhine (éd.), Mondes Clos. Les îles », Anabases [En ligne], 24 | 2016, mis en ligne le 15 novembre 2016, consulté le 23 septembre 2020. URL : http:// journals.openedition.org/anabases/5735; DOI : https://doi.org/10.4000/anabases.5735

Ce document a été généré automatiquement le 23 septembre 2020.

(C) Anabases 


\title{
Daniel Barbu, Nicolas Meylan, Youri Volokhine (éd.), Mondes Clos. Les îles
}

\author{
Élodie Guillon
}

\section{RÉFÉRENCE}

Daniel Barbu, Nicolas Meylan, Youri Volokhine (éd.), Mondes Clos. Les îles, Gollion, Infolio éditions, 2015, 274 p.-[XXV] p. de pl.

29 euros / isbn 978-2-88474-354-9

1 Daniel Barbu, Nicolas Meylan et Youri Volokhine proposent un volume consacré aux îles, le deuxième d'une série sur les «mondes clos ", qui se définissent, à travers les époques, par le tracé, la frontière, voire l'isolat. Les éditeurs présentent ce volume collectif, qui comporte treize contributions, précédées d'une introduction, comme une réflexion pluridisciplinaire sur les rapports que les hommes entretiennent avec ces morceaux de terre, qu'ils soient réels ou encore fictifs. Les îles, en effet, sont le lieu de projection de fantasmes, de rêves, mais également le lieu d'expériences utopiques et de désillusions. Pour étudier ces rapports, le cadre spatio-temporel retenu est large, du Pacifique à l'Inde, en passant par l'Afrique et les îles britanniques, de l'Antiquité à l'époque contemporaine.

2 Sur les cinq parties structurant le volume, la première, intitulée «Dans les mers du sud", est consacrée aux représentations projetées sur les îles du Pacifique et aux expériences qui en sont faites entre le xvi ${ }^{\mathrm{e}}$ et le xviii ${ }^{\mathrm{e}}$ siècle. Dominique Barbe analyse le rapprochement fait entre ces îles et le Paradis, dans ses nuances catholiques et protestantes, pour présenter le contexte intellectuel et culturel des colons missionnaires et laïcs, partis s'établir dans ces archipels, confrontés à une réalité en décalage avec leurs représentations et à la nécessité, finalement, de construire leur propre paradis. Jan Blanc se concentre plus spécifiquement sur le peintre William Hodges, parti avec Thomas Cook. Cette fois, l'expérience de l'île et la nouveauté se confrontent avec la nécessité de la représenter pour expliquer les découvertes en 
Europe. L'expérience des voyages et des îles, la curiosité et la liberté qui les motivent, viennent nourrir une réflexion sur la peinture de paysage, qui se cherche alors, en Angleterre, de nouveaux modèles.

3 La deuxième partie s'intéresse aux « Îles des confins » telles qu'elles sont évoquées et représentées dans des textes sanscrits et cunéiformes. Les îles, souvent résidences des dieux, y sont séparées par un élément liquide, et ne peuvent être atteintes que difficilement, par quelques-uns seulement. Philippe Bornet montre que les toponymes sanscrits «île dorée » et "île blanche » font écho à des modèles cosmologiques complexes, sans localisation géographique. Pourtant, ils sont investis plus tardivement d'une dimension territoriale, qui participe d'une réflexion spéculative liée à des préoccupations contemporaines: les relations Inde-Angleterre au xix ${ }^{\mathrm{e}}$ siècle ou la question du rôle de l'Inde dans l'histoire universelle, posée au début $\mathrm{du} \mathrm{xx}^{\mathrm{e}}$ siècle. Anne-Caroline Rendu Loisel examine ensuite les îles mésopotamiennes des confins, qui rappellent également une conception du monde: un continent gouverné par les Babyloniens et les Assyriens, un océan cosmique et des îles, au loin, espace liminal entre le connu et l'inconnu, entre l'accessible et l'inaccessible.

Cette partie annonce la suivante, "Mythologie des îles", qui démontre que ces dernières sont le lieu privilégié de l'élaboration de récits. Doralice Fabiano met en évidence la lecture genrée de Délos que faisaient les Anciens, qui à la fois rappelait la place des filles et des femmes dans la construction sociale grecque, et participait d'un système complexe d'appréhension de l'espace ouvrant une interaction rituelle avec ce dernier - grâce à l'identification Délos-Astérie. Puis Philippe Borgeaud présente le « livre des îles » de la Bibliothèque historique de Diodore, support d'un récit évhémériste expliquant l'hégémonie romaine et d'une vision idéalisée de son île d'origine, la Sicile, terre de valeurs qui ne sont pas le privilège des vainqueurs. Enfin, Philippe Matthey, en prenant pour exemple les récits légendaires irlandais, riches d'îles merveilleuses et de pommes magiques, montre combien les traditions classiques et bibliques portant sur l'insularité, l'image des Îles Fortunées, du jardin des Hespérides - transformé en île dans les textes de la fin de l'Antiquité - et de l'Eden se croisent et s'enrichissent mutuellement.

Dans «Dérives insulaires ", Frank Lestringant met en évidence, à travers des cosmographies du $\mathrm{XVI}^{\mathrm{e}}$ siècle, un temps des îles, quand le monde en expansion est perçu comme un émiettement des terres en un archipel indénombrable, avant de se recomposer avec la progression des découvertes. L'Islande du xiiie siècle est ensuite l'objet de la contribution de Nicolas Meylan, qui analyse ses rapports avec la Norvège, alors en pleine expansion. On regrette toutefois de ne pas voir l'insularité islandaise mise au cœur de cette analyse politique. Angela Benza propose, pour clore cette partie, d'analyser le rapport complexe d'Élizabeth Ire à son île, dépassant de loin la simple possession. Elle met en lumière les interactions entre la reconstruction du pouvoir monarchique et la définition iconographique de l'Angleterre. L'île fixe alors son identité, entre isolement et perméabilité, entre particularisme anglais et interaction avec le reste du monde.

6 Enfin, Neil Forsyth boucle le tour des îles dans un "Retour dans les mers chaudes", avec un écho géographique et conceptuel à la première partie du volume, grâce à une lecture à rebours du poème d'Andrew Marvell, Bermudas (1653-54), trop souvent lu comme un simple topos littéraire associant paradis et île. 
7 On attendrait finalement un mot de conclusion sur ce riche parcours, qui aborde plusieurs notions fécondes comme celles de limite, de perméabilité ou encore de mobilité. Peut-être cette absence tient-elle à la diversité des approches et des conclusions liées au monde clos qu'est l'île, que le choix d'une réflexion pluridisciplinaire a d'ailleurs parfaitement mise en lumière? S'il manque quelques rappels pour les néophytes sur certains contextes ou notions, on retiendra surtout que l'insularité est un concept pertinent pour une anthropologie spatiale, qui analyse les rapports de l'homme à son environnement tant physique que culturel et symbolique.

\section{AUTEURS}

\section{ÉLODIE GUILLON}

Université Toulouse-Jean Jaurès (UT2J)

eguillon31@gmail.com 